Original Paper http://ajol.info/index.php/ijbcs http://indexmedicus.afro.who.int

\title{
Efficacité des anthelminthiques usuels contre les strongles digestifs chez les ovins au centre de la Côte d'ivoire
}

\author{
Alain Gérard Ambroise APALA ${ }^{1 *}$, Amoin Marie Amélie Clarisse KOMOIN-OKA ${ }^{2}$, \\ Kouakou Rufin ASSARE ${ }^{1}$, Gédéon AMIAN ${ }^{3}$ et Kouakou Eliezer N'GORAN ${ }^{1}$
}

${ }^{1}$ Laboratoire de Zoologie Biologie Animale, UFR Biosciences, Université Felix Houphouët Boigny, 01 BP V 34 Abidjan, Côte d'Ivoire.

${ }^{2}$ Laboratoire Central Vétérinaire de Bingerville, BP 206 Bingerville, Côte d'Ivoire.

${ }^{3}$ Agence Nationale d'Appui au Développement Rural BP V 704 Abidjan, Côte d'Ivoire.

*Auteur correspondant, E-mail : apalaspike2005@yahoo.fr /Tel :0022549434180

\section{RESUME}

Les données recueillies lors des études menées par certains chercheurs ont donné des prévalences élevées à l'infestation des strongles dans certaines régions de la Côte d'Ivoire, laissant soupçonner une diminution de l'efficacité des anthelminthiques dans les élevages ovins. Nous avons supposé que les moyens de lutte contre les strongles deviennent moins efficaces. C'est donc pour confirmer cette baisse de l'efficacité des traitements anthelminthiques, en déterminer les causes et proposer des solutions pour enrayer ce phénomène que cette étude a été réalisée. Elle s'est déroulée dans 12 élevages répartis dans 8 localités du centre de la Cote d'Ivoire. Elle visait à évaluer par des tests in vivo de réduction de l'excrétion fécale des œufs (FECRT), la sensibilité des strongles gastro intestinaux aux anthelminthiques usuels, afin de vérifier l'apparition de cas de résistance. La molécule la plus utilisée était l'Albendazole, suivie du Lévamisole, puis de l'Ivermectine. L'Ivermectine a été testée dans un seul élevage, le Lévamisole dans 4 élevages et l'Albendazole dans 10 élevages. Les analyses coprologiques ont permis l'identification d'œufs de strongles, de Trichuris sp, de Strongyloides sp, de Moniezia sp et d'ookystes du genre Eimeria. Avec l'Ivermectine, le taux de réduction de l'excrétion fécale (FECR) des œufs de strongles a été de $100 \%$ dans le seul élevage où il a été testé. Avec le Lévamisole, le FECR a été de $100 \%$ dans 2 élevages et il était compris entre 92 et 99\% dans les 2 autres élevages. Avec l'Albendazole, le FECR a été de $100 \%$ dans 7 élevages; il était compris entre 94 et $99 \%$ dans 2 élevages, et entre 70 et $96 \%$ dans un élevage. Des autopsies ont permis d'identifier les espèces de strongles; il s'agissait d'Haemonchus sp, Trichostrongylus sp, Cooperia sp et Oesophagostomum sp. Les animaux autopsiés provenant des élevages où le FECR était inférieur à 100\%, hébergeaient un nombre de vers plus important que les animaux des autres élevages. Ce travail a permis de démontrer que la résistance aux anthelminthiques ne s'est pas encore installée dans les élevages ovins au centre de la Côte d'Ivoire. Un tel travail doit être étendu à l'échelle national avec des tests in vitro, afin de mieux cerner l'état des lieux, et par la même occasion sensibiliser l'ensemble des éleveurs sur les différentes pratiques à adopter afin de retarder voire empêcher la mise en place de la résistance dans nos différentes fermes en Côte d'Ivoire.

(C) 2020 International Formulae Group. All rights reserved.

Mots clés : Efficacité, anthelminthiques, strongles digestifs, ovins, Côte d'Ivoire. 


\title{
Efficacy of common anthelmintics against digestive strongles in sheep in central of Côte d'Ivoire
}

\begin{abstract}
The data collected during the studies carried out by some researchers gave high prevalence to the infestation of strongles in certain regions of Côte d'Ivoire, suggesting a decrease in the efficacy of anthelmintics in sheep farming. We have assumed that the means of combating strongles become less effective. This study was therefore carried out to confirm this drop in the effectiveness of anthelmintic treatments, to determine the causes and to propose solutions to curb this phenomenon. It took place in 12 farms spread over 8 localities in the center of Côte d'Ivoire. It aimed to evaluate, by in vivo tests for the reduction of faecal excretion of eggs (FECRT), the sensitivity of gastrointestinal strongyles to the usual anthelmintics, in order to check the appearance of cases of resistance. The most used molecule was Albendazole, followed by Levamisole and then Ivermectin. Ivermectin was tested in a single farm, Levamisole in 4 farms and Albendazole in 10 farms. Coprology analyzes identified eggs of strongyles, Trichuris sp, Strongyloides sp, Moniezia sp and Oookies of the genus Eimeria. With Ivermectin, the rate of fecal excretion reduction (FECR) of strongle eggs was $100 \%$ in the one farm where it was tested. With Levamisole, the FECR was $100 \%$ in 2 farms and it was between 92 and $99 \%$ in the 2 other farms. With Albendazole, the FECR was 100\% in 7 farms; it was between 94 and $99 \%$ in 2 farms, and between 70 and $96 \%$ in a farm. Autopsies identified species of strongyles; these were Haemonchus sp, Trichostrongylus sp, Cooperia sp and Oesophagostomum sp. Autopsied animals from farms where the FECR was less than $100 \%$ harbored a larger number of worms than animals from other farms. This work has made it possible to demonstrate that resistance to anthelmintics has not yet taken hold in sheep farming in the center of Côte d'Ivoire. Such work must be extended to the national level with in vitro tests, in order to better understand the inventory, and at the same time make all the breeders aware of the different practices to adopt in order to delay or even prevent the resistance set up in our different farms in Ivory Coast.
\end{abstract}

(C) 2020 International Formulae Group. All rights reserved.

Keywords: Efficacy, anthelmintics, digestive strongles, sheep, Côte d'Ivoire.

\section{INTRODUCTION}

Les strongles gastro-intestinaux (SGI) sont des parasites responsables de pathologies graves et de pertes économiques importantes dans les élevages de ruminants. Les strongyloses sont fréquentes en élevage des petits ruminants, et constituent donc des pathologies majeures (Emanfo et al., 2015). Pour lutter contre elles, les éleveurs ont recours aux anthelminthiques. Malheureusement leur utilisation répétée a permis aux parasites de développer une résistance irréversible à la majorité des familles de ces molécules (Kaplan, 2004 ; Cabaret, 2012). Ce phénomène de résistance, qui touche presque toutes les espèces de strongles, s'étend de plus en plus à l'ensemble des régions du monde, notamment chez les petits ruminants et les équidés (Zoubli, 2006 ; Jacquiet et al., 2009).

Dans les régions humides d'Afrique de l'ouest et plus précisément en Côte d'Ivoire, les prévalences d'infestations des ruminants par les SGI sont d'au moins 95\%. Les infestations se déroulent toute l'année avec cependant des pics liés à la pluviométrie (Achi et al., 2003 ; Touré et al., 2006 ; Emanfo et al., 2015). Pour lutter contre le parasitisme chez les ovins, les éleveurs les vermifugent d'une façon systématique et fréquente.

L'objectif de cette étude est d'évaluer, par des tests in vivo, la sensibilité des SGI aux anthelminthiques couramment utilisés par les éleveurs afin de mettre en évidence d'éventuels cas de résistance dans les élevages ovins du centre de la Cote d'Ivoire et recommander une amélioration des stratégies de traitement.

\section{MATERIEL ET METHODES \\ Zone d'étude}

Cette étude a été réalisée au centre de la Côte d'Ivoire, d'août 2017 à décembre 2018. Elle a débuté par une enquête par questionnaire réalisée dans 31 élevages ovins localisés dans les régions du Gbeke, du Hambol et du Bélier. 
Douze élevages situés dans 8 localités de la région du Bélier ont ensuite été sélectionnés pour des tests de résistances aux anthelminthiques (Figure 1). La zone centre de la Cote d'Ivoire concentre environ $40 \%$ du cheptel ovin national, estimé à 1836357 animaux en 2017 (FAO, 2019). Elle est caractérisée par un relief relativement peu accidenté avec des plateaux étagés d'une altitude de 200 à $500 \mathrm{~m}$, séparés par des talus de faible hauteur (10 à $30 \mathrm{~m}$ ); la végétation comporte des lambeaux de forêts et de larges mailles de savanes séparées par des forêts galeries (Konaté et Kampmann, 2010). Le climat est de type équatorial de transition atténué avec 2 saisons sèches et 2 saisons pluvieuses. Pendant la période d'étude, les températures ont été en moyenne de $27,5^{\circ} \mathrm{C}$ et la pluviométrie de $86,25 \mathrm{~mm}$ (Figure 2).

Les 31 fermes, où a été réalisée l'enquête par questionnaire, ont été identifiées avec l'aide des agents de l'Agence Nationale d'Appui au Développement Rural (ANADER). Le questionnaire avait pour objectif de connaitre les pratiques des éleveurs dans le suivi de leurs animaux. Il a porté sur les axes suivants: l'alimentation, l'abreuvement, l'hygiène, le suivi des animaux, la prophylaxie et le traitement du parasitisme digestif.

Les 12 élevages retenus pour le test de résistance aux anthelminthiques ont été sélectionnés sur les critères suivants : l'accessibilité de l'élevage par un véhicule, la taille du troupeau (au moins 25 animaux), la disponibilité d'un parc (pour faciliter la contention des animaux), la fréquence de déparasitage interne (au moins une fois par an) et la volonté de participation de l'éleveur.

\section{Les animaux}

En fonction de l'effectif du cheptel, des lots de 25 ovins au minimum, ont été constitués de façon aléatoire, en excluant cependant les femelles gestantes. Les tests de résistance aux anthelminthiques ont ainsi été réalisés sur 550 animaux, mâles et femelles, de tout âge, répartis dans les 3 systèmes de production : 198 (soit 36\%) appartenant au système traditionnel, 159 (soit 28,9\%) appartenant au système amélioré ou moderne semi-intensif et 193 (soit
$35,1 \%$ ) appartenant au système amélioré ou moderne intensif. Ces animaux n'avaient pas été vermifugés depuis au moins 2 mois. Ils ont été bouclés, s'ils ne l'étaient pas déjà, avant de procéder aux prélèvements fécaux individuels.

\section{Les tests de résistance in vivo}

Le test utilisé a été le test de réduction de l'excrétion fécale des œufs (FECRT) (Coles et al., 1992). Au moins 10 grammes de matières fécales ont été prélevés directement dans le rectum de chaque ovin. Les animaux ont été ensuite pesés, afin de calculer la dose individuelle d'anthelminthique à administrer à chacun. Les produits anthelminthiques utilisés pour les tests sont ceux couramment utilisés dans l'élevage: Albendazole, Lévamisole et/ou Ivermectine. En fonction du nombre de molécules utilisées et de l'effectif du cheptel, 2 ou 3 lots d'ovins étaient constitués, y compris le lot témoin (Tableau 1). L'administration de l'anthelminthique a été faite exclusivement par voie orale, à J0. Huit jours après la collecte des fèces et l'administration de l'anthelminthique, soit $\mathrm{J} 8$, les ovins ont été soumis à une seconde collecte de matières fécales. Les prélèvements des matières fécales ont été conservés sous glace et acheminés dans un délai maximum de 24 heures au laboratoire. Dès l'arrivée au laboratoire, les examens coprologiques ont été réalisés selon la technique de Mac Master modifiée (Coles et al., 1992), avec une solution saturée de chlorure de sodium d'une densité de 1,2. La charge parasitaire des strongles des animaux a ainsi pu être estimée en fonction du nombre d'œufs par gramme (opg) de matière fécale. Le taux de réduction de l'excrétion fécale (fecr) a été calculé selon les formules suivantes :

- Fecr1 $(\%)=1-($ MT2/MC2) x 100, selon Coles et al. (1992);

- Fecr2 $(\%)=1-($ MT2/MT1) x 100, selon Kochapakdee et al. (1995);

- Fecr3 $(\%)=1-[(\mathrm{MT} 2 / \mathrm{MT} 1) \times(\mathrm{MC} 1 / \mathrm{MC} 2)]$ x 100, selon Dash et al. (1988);

- Fecr4 $(\%)=1-(\mathrm{MT} 2 / \mathrm{MC} 1) \times 100$, selon Powers et al. (1982);

Où MT1 et MT2 sont les moyennes des opg des animaux traités respectivement à $\mathrm{J} 0$ et à $\mathrm{J} 8$; et $\mathrm{MC} 1$ et MC2 celles des animaux non traités. 


\section{Autopsie et recherche de strongles digestifs} adultes

A l'issue du FECRT, un animal de chaque lot a été sacrifié pour vérifier la disparition des strongles suite au traitement. En cas de persistance des strongles, les espèces présentes ont été identifiées. Un total de 15 ovins ont ainsi été abattus pour autopsie, provenant de 11 élevages, l'un des éleveurs (élevage $n^{\circ} 1$ ) ayant refusé de sacrifier des animaux. Après abattage de l'animal, les compartiments digestifs (caillette, intestin grêle et gros intestin) ont été isolés par des ligatures. Chaque compartiment a été ouvert et son contenu a été passé à travers un tamis de $200 \mu \mathrm{m}$ de diamètre. Les résidus du tamis ont été recueillis dans un bocal et dilués, la suspension obtenue a été entièrement examinée, par de petites fractions, sous la loupe ( $\mathrm{G} \times 20$ ou $\times 40$ ), afin de rechercher d'éventuels parasites.

\section{Exploitation des données et analyses statistiques}

Les données ont été analysées avec le logiciel STATA version 13.1 (Stata corporation College Station, United states of America).

Les résultats du Fecr ont été interprétés suivant les recommandations de la World Association for The Advancement of veterinary Parasitology (Coles et al., 1992). Si le pourcentage de réduction est inférieur à $95 \%$, une résistance à l'anthelminthique est suspectée; s'il est inférieur à $90 \%$, une résistance existe.

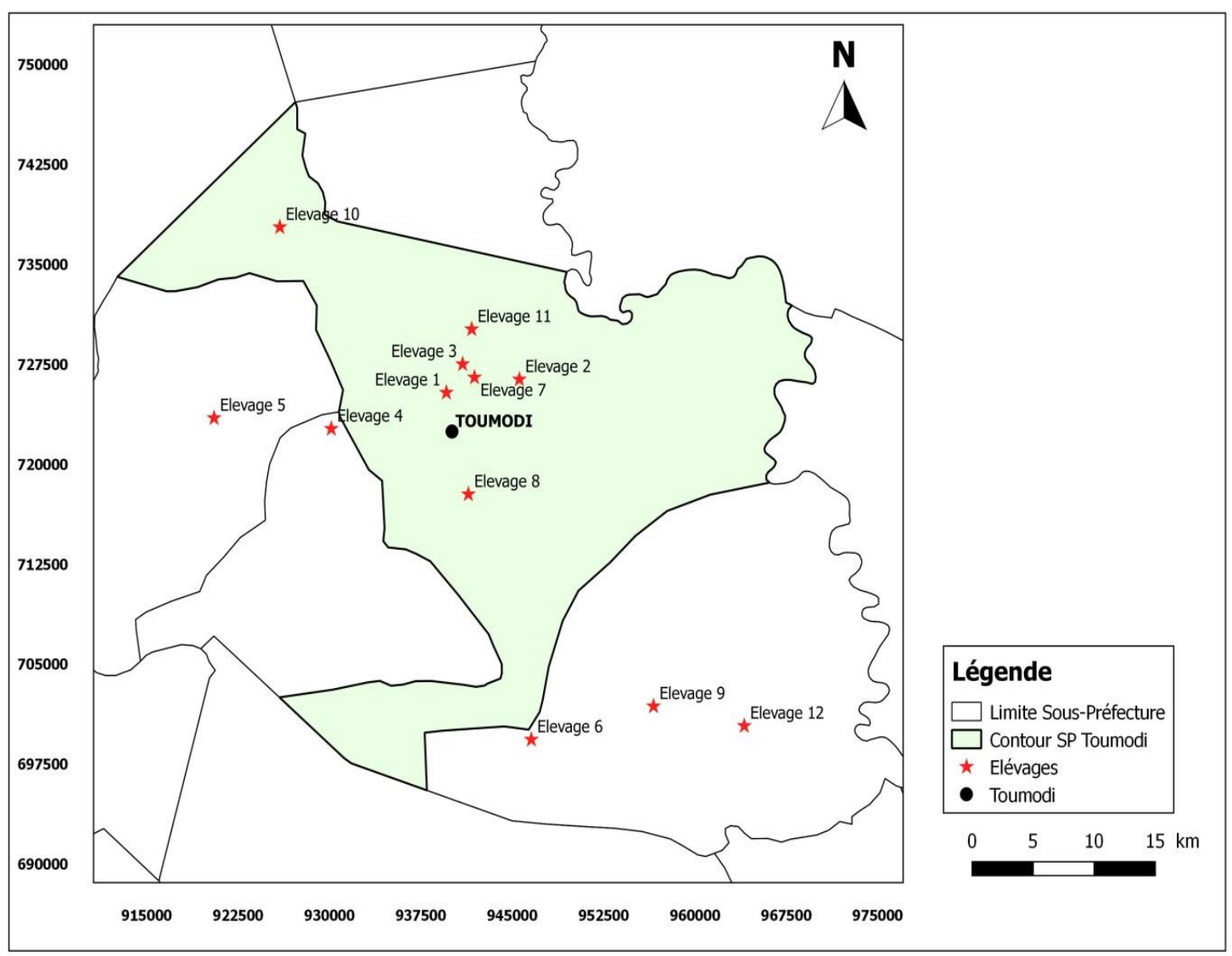

Figure 1 : zone d'étude. 
Tableau 1 : Répartition des animaux par système de production.

\begin{tabular}{|c|c|c|c|c|c|c|c|c|c|c|}
\hline \multirow{2}{*}{$\begin{array}{l}\text { Systèmes de } \\
\text { production }\end{array}$} & \multirow{2}{*}{$\begin{array}{l}\text { Numéro } \\
\text { d'élevage }\end{array}$} & \multirow[t]{2}{*}{ Localités } & \multirow{2}{*}{$\begin{array}{l}\text { Age de } \\
\text { la ferme }\end{array}$} & \multicolumn{3}{|c|}{ Nombre de traitement / molécule / an } & \multirow{2}{*}{$\begin{array}{c}\text { Date du } \\
\text { dernier } \\
\text { déparasitage }\end{array}$} & \multirow{2}{*}{$\begin{array}{c}\text { Date du } \\
\text { prélèvement }\end{array}$} & \multirow{2}{*}{$\begin{array}{c}\begin{array}{c}\text { Nombre } \\
\text { d'animaux } \\
\text { prélevés }\end{array} \\
(\mathbf{n}=\mathbf{5 5 0})\end{array}$} & \multirow{2}{*}{$\begin{array}{c}\text { Répartition } \\
\text { des animaux } \\
\text { prélevés } \\
\text { selon l'âge }\end{array}$} \\
\hline & & & & Albendazole & Levamisole & Ivermectine & & & & \\
\hline \multirow[t]{6}{*}{ Traditionnel } & 1 & Toumodi & 10 ans & 6 & 0 & 0 & juil-17 & janv-18 & 35 & \multirow{6}{*}{$\begin{array}{c}0-1 \text { an }: 57 \\
1-2 \text { ans }: 66 \\
>2 \text { ans }: 75\end{array}$} \\
\hline & 3 & Toumodi & 9 ans & 6 & 6 & 0 & nov-17 & janv-18 & 35 & \\
\hline & 4 & Yobouekro & 12 ans & 1 & 0 & 5 & oct-17 & févr-18 & 35 & \\
\hline & 5 & Kplessou & 19 ans & 0 & 0 & 0 & $\begin{array}{c}\text { pas de } \\
\text { déparasitage } \\
\text { massif* }\end{array}$ & févr-18 & 35 & \\
\hline & 6 & Moronou & 2 ans & 4 & 0 & 0 & août-17 & févr-18 & 28 & \\
\hline & 10 & Ndoukahakro & 6 ans & 0 & 2 & 0 & nov-17 & mars-18 & 30 & \\
\hline \multirow{3}{*}{$\begin{array}{c}\text { Semi- } \\
\text { traditionnel }\end{array}$} & 8 & Blé & 5 ans & 6 & 6 & 0 & déc-17 & févr-18 & 85 & \multirow{3}{*}{$\begin{array}{c}0-1 \text { an }: 45 \\
1-2 \text { ans }: 45 \\
>2 \text { ans }: 69\end{array}$} \\
\hline & 9 & Kpouèbo & 2 ans & 4 & 0 & 0 & janv-18 & mars-18 & 46 & \\
\hline & 11 & Abli & 5 ans & 12 & 0 & 0 & févr-18 & avr-18 & 28 & \\
\hline \multirow[t]{3}{*}{ Moderne } & 2 & Toumodi & 14 ans & 4 & 2 & 0 & nov-17 & janv-18 & 67 & \multirow{3}{*}{$\begin{array}{c}0-1 \text { an }: 41 \\
1-2 \text { ans }: 83 \\
>2 \text { ans : } 69\end{array}$} \\
\hline & 7 & Toumodi & 20 ans & 4 & 4 & 4 & janv-18 & mars-18 & 81 & \\
\hline & 12 & Kpouèbo & 10 ans & 6 & 6 & 0 & févr-18 & avr-18 & 45 & \\
\hline
\end{tabular}




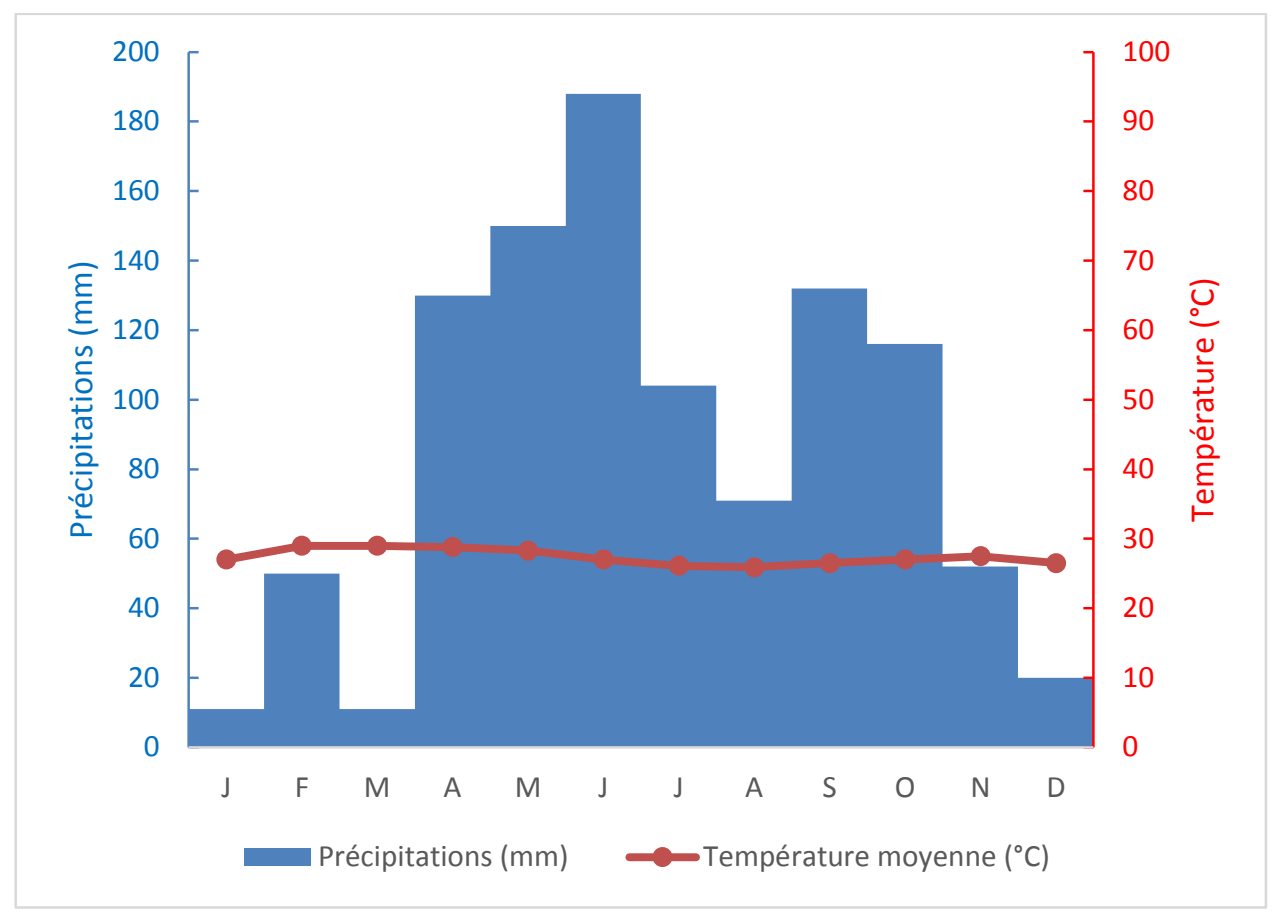

Figure 2: Températures moyennes et pluviométrie dans la zone de Toumodi durant l'année 2018.

\section{RESULTATS}

Les 550 animaux de l'étude comprennent 146 mâles et 404 femelles. Vingtsix pour cent d'entre eux sont âgés de moins d'un an ; $35,3 \%$ ont entre 1 et 2 ans et $38,7 \%$ ont plus de 2 ans (Tableau 1).

Les analyses coprologiques réalisées à J0 ont permis d'identifier des œufs de strongles, de Trichuris sp, de Strongyloides sp, de Moniezia $s p$ et des ookystes de coccidies du genre Eimeria. Les charges parasitaires en œufs de strongles étaient comprises entre 0 et 13500 opg à $\mathrm{J} 0$, avec une moyenne de 280 opg (IC : 202,9 - 355,7). Après traitement, les charges parasitaires n'étaient plus comprises qu'entre 0 et 200 opg, avec une moyenne de 5,2 (IC : 1,9 - 10,2) (Tableau 2).

L'ivermectine n'a été testée que dans un seul élevage; l'excrétion fécale des œufs a présenté un taux de réduction de $100 \%$; quelle que soit la méthode de calcul (Tableau 3). Le lévamisole a été testé seul dans un élevage $\left(\mathrm{n}^{\circ} 10\right)$; le taux de réduction de l'excrétion fécale y a varié de $95,3 \%$ à $99,0 \%$. L'albendazole a été testé seul dans 7 élevages : dans 5 élevages, le Fecr était de 100\%; dans les 2 autres élevages, il était compris entre 96,4 et $98,7 \%$ (élevage $n^{\circ} 6$ ), et entre $70,8 \%$ et $98,8 \%$ (élevage $n^{\circ} 11$ ). Dans 3 élevages, le lévamisole et l'albendazole ont été testés en même temps ; si dans 2 élevages, le fecr a été de $100 \%$ pour les 2 molécules, dans le troisième élevage $\left(n^{\circ} 8\right)$, il présente un pourcentage de réduction situé entre 92,5 et $96,9 \%$ pour l'albendazole et 94,2 et $99,1 \%$ pour le lévamisole (Tableau 3).

Dans 11 élevages sur 12, les ovins reçoivent des traitements de masse contre les strongles digestifs de 2 à 12 fois par an, tandis que dans l'élevage $\mathrm{n}^{\circ} 5$, situé à Kpouèbo, l'éleveur fait des traitements individuels ne portant que sur les animaux apparaissant mal en point (Tableau 1). Dans les élevages $n^{\circ} 8$ et $\mathrm{n}^{\circ} 11$, où le Fecr est inférieur à $100 \%$, les vermifugations sont effectuées chaque mois, avec l'albendazole seul dans l'élevage $n^{\circ} 11$, et avec le lévamisole et l'albendazole utilisés en alternance dans l'élevage $n^{\circ} 8$. Dans les élevages $\mathrm{n}^{\circ} 6$ et $\mathrm{n}^{\circ} 10$, où le Fecr est également inférieur à $100 \%$, les vermifugations sont moins fréquentes, respectivement 2 fois par an et tous les 2 mois. 
Les autopsies ont permis d'observer les formes adultes de toutes les espèces d'helminthes détectées par la coproscopie, et d'identifier les espèces de strongles présentes. Il s'agissait notamment d'Haemonchus sp, Trichostrongylus sp, Cooperia sp et Oesophagostomum sp. Dans les élevages où le Fecr est de $100 \%$, soit aucun ver adulte n'a été observé dans le tube digestif des animaux (cas de trois élevages : $\mathrm{n}^{\circ} 3,4$ et 5 ), soit leur nombre était faible (Tableau 4). Par contre, dans les élevages où le Fecr est inférieur à $100 \%$, de nombreux helminthes ont été récoltés, particulièrement dans les élevages $n^{\circ} 8$ (jusqu'à 718 vers) et $n^{\circ} 11$ (jusqu'à 1680 vers).

Tableau 2 : Opg moyen des strongles avant et après traitement.

\begin{tabular}{lcccc}
\hline & Effectif & OPG Moyen & $\begin{array}{c}\text { Erreur } \\
\text { standard }\end{array}$ & IC 95\% \\
\hline Avant traitement & 550 & 279,77 & 38,89 & $202,86-355,68$ \\
\hline Après traitement & 495 & 5,23 & 1,77 & $1,95-10,24$ \\
\hline
\end{tabular}

Tableau 3 : Efficacité des molécules anthelminthiques contre les SGI chez des ovins.

\begin{tabular}{lccccccccc}
\hline Molécule testée & Fermes & $\begin{array}{c}\text { Mc1 } \\
(\mathbf{n})\end{array}$ & $\begin{array}{c}\text { Mc2 } \\
(\mathbf{n})\end{array}$ & $\begin{array}{c}\text { Mt1 } \\
(\mathbf{n})\end{array}$ & $\begin{array}{c}\text { Mt2 } \\
(\mathbf{n})\end{array}$ & $\begin{array}{c}\text { Fecr } \\
\mathbf{1}\end{array}$ & $\begin{array}{c}\text { Fecr } \\
\mathbf{2}\end{array}$ & $\begin{array}{c}\text { Fecr } \\
\mathbf{3}\end{array}$ & $\begin{array}{c}\text { Fecr } \\
\mathbf{4}\end{array}$ \\
\hline Ivermectine & 4 & 380 & 350 & 360 & 0 & 100 & 100 & 100 & 100 \\
& & $(5)$ & $(5)$ & $(30)$ & $(29)$ & & & & \\
\hline Lévamisole & 10 & 250 & 750 & 157 & 7,4 & 99,0 & 95,3 & 98,4 & 97,0 \\
& & $(5)$ & $(5)$ & $(27)$ & $(27)$ & & & & \\
\hline Albendazole & 1 & 320 & 610 & 667 & 0 & 100 & 100 & 100 & 100 \\
& & $(5)$ & $(5)$ & $(30)$ & $(28)$ & & & & \\
& 3 & 170 & 500 & 177 & 0 & 100 & 100 & 100 & 100 \\
& & $(5)$ & $(3)$ & $(30)$ & $(30)$ & & & & \\
& 5 & 260 & 200 & 125 & 0 & 100 & 100 & 100 & 100 \\
& & $(5)$ & $(5)$ & $(30)$ & $(30)$ & & & & \\
& & 400 & 350 & 938 & 12,5 & 96,4 & 98,7 & 98,5 & 96,9 \\
& & $(3)$ & $(3)$ & $(25)$ & $(25)$ & & & & \\
& & 0 & 75 & 250 & 0 & 100 & 100 & 100 & 100 \\
& & $(5)$ & $(4)$ & $(41)$ & $(41)$ & & & & \\
& & 100 & 850 & 280 & 29,2 & 96,5 & 89,6 & 98,8 & 70,8 \\
& $(5)$ & $(4)$ & $(25)$ & $(24)$ & & & & \\
& 11 & 100 & 470 & 450 & 0 & 100 & 100 & 100 & 100 \\
& $(5)$ & $(5)$ & $(40)$ & $(40)$ & & & & \\
& & & & & & & & &
\end{tabular}


A. G. A. APALA et al. / Int. J. Biol. Chem. Sci. 14(2): 378-389, 2020

\begin{tabular}{|c|c|c|c|c|c|c|c|c|c|c|}
\hline \multirow{5}{*}{$\begin{array}{l}\text { Albendazole } \\
+ \\
\text { Lévamisole }\end{array}$} & \multirow[t]{2}{*}{2} & Alb. & 150 & 200 & 225 & 0 & 100 & 100 & 100 & 100 \\
\hline & & Lév. & $\begin{array}{c}150 \\
(5)\end{array}$ & $\begin{array}{l}200 \\
(5)\end{array}$ & $\begin{array}{l}893 \\
(30)\end{array}$ & $\begin{array}{c}0 \\
(30)\end{array}$ & 100 & 100 & 100 & 100 \\
\hline & \multirow[t]{2}{*}{7} & Alb. & $\begin{array}{c}300 \\
(5)\end{array}$ & $\begin{array}{l}500 \\
(5)\end{array}$ & $\begin{array}{l}170 \\
(41)\end{array}$ & $\begin{array}{c}0 \\
(41)\end{array}$ & 100 & 100 & 100 & 100 \\
\hline & & Lév. & $\begin{array}{c}300 \\
(5)\end{array}$ & $\begin{array}{l}500 \\
(5)\end{array}$ & $\begin{array}{l}150 \\
(35)\end{array}$ & $\begin{array}{c}0 \\
(35)\end{array}$ & 100 & 100 & 100 & 100 \\
\hline & 8 & Alb. & $\begin{array}{c}200 \\
(5)\end{array}$ & $\begin{array}{c}100 \\
(5)\end{array}$ & $\begin{array}{l}286 \\
(40)\end{array}$ & $\begin{array}{c}8,8 \\
(40)\end{array}$ & 92,5 & 96,9 & 93,9 & 95,6 \\
\hline
\end{tabular}

Tableau 4 : Genre des Helminthes identifiés par autopsie.

\begin{tabular}{|c|c|c|c|c|c|c|c|c|c|c|}
\hline \multirow{2}{*}{$\begin{array}{l}\text { Molécule } \\
\text { testée }\end{array}$} & \multirow{2}{*}{\multicolumn{2}{|c|}{ Fermes }} & \multicolumn{4}{|c|}{ Genre des Strongles } & \multicolumn{2}{|c|}{ Autres nématodes } & \multirow{2}{*}{$\begin{array}{l}\text { Cestodes } \\
\text { Moniezia }\end{array}$} & \multirow{2}{*}{$\begin{array}{c}\text { Nombre } \\
\text { total de } \\
\text { nématod } \\
\text { es/ } \\
\text { animal }\end{array}$} \\
\hline & & & Haemonch. & Trichos. & Cooperia & Oesophag. & Trichuris & Strongyl. & & \\
\hline Ivermectine & 4 & & 0 & 0 & 0 & 0 & 0 & 0 & présence & 0 \\
\hline Lévamisole & 10 & & 191 & 41 & 0 & 0 & 0 & 0 & présence & 232 \\
\hline \multirow[t]{6}{*}{ Albendazole } & 3 & & 0 & 0 & 0 & 0 & 0 & 0 & présence & 0 \\
\hline & 5 & & 0 & 0 & 0 & 0 & 0 & 0 & présence & 0 \\
\hline & 6 & & 10 & 0 & 1 & 0 & 3 & 375 & absence & 389 \\
\hline & 9 & & 113 & 3 & 0 & 2 & 0 & 0 & absence & 118 \\
\hline & 11 & & 1637 & 0 & 43 & 0 & 0 & 0 & présence & 1680 \\
\hline & 12 & & 62 & 0 & 0 & 0 & 1 & 0 & présence & 63 \\
\hline \multirow{6}{*}{$\begin{array}{l}\text { Albendazole } \\
+ \text { Lévamisole }\end{array}$} & 2 & Alb. & 54 & 0 & 0 & 0 & 0 & 0 & absence & 54 \\
\hline & & Lév. & 14 & 0 & 0 & 0 & 0 & 0 & présence & 14 \\
\hline & 7 & Alb. & 123 & 0 & 0 & 0 & 0 & 1 & présence & 124 \\
\hline & & Lév. & 0 & 0 & 0 & 0 & 0 & 0 & présence & 0 \\
\hline & 8 & & 421 & 26 & 271 & 0 & 0 & 0 & présence & 718 \\
\hline & & & 475 & 4 & 52 & 0 & 1 & 0 & présence & 532 \\
\hline
\end{tabular}




\section{DISCUSSION}

Il est rapporté de plus en plus des cas de résistance aux anthelminthiques dans les élevages (Zouiten, 2006; Tanguy, 2011; Eischtadt, 2017). La résistance à une substance se définit comme étant le développement de la capacité d'individus à tolérer des doses de toxique qui seraient mortelles pour la majorité des individus d'une population normale de la même espèce (FAO, 2004). Selon la World Association for the Advancement of Veterinary Parasitology (WAAVP), on parlera de suspicion de résistance quand moins de $95 \%$ des individus sont éliminés par les produits administrés. Okombe et Pougombo (2013) ramènent ce seuil à $90 \%$.

Quand une résistance est déclarée chez les strongles, les niveaux d'OPG restent en général élevés après l'administration des anthelminthiques (Boulkaboul, 2006). Dans cette étude, l'OPG après traitement est resté très globalement faible. Cela dénote d'une bonne efficacité des produits testés, comme concluent les auteurs Kouakou et al.,(2010) Sacramento et al., (2010) et Sidi et al., (2015) lors de leur étude visant à évaluer respectivement l'effet antiparasitaire des inflorescences de Thonningia sanguinea (Balanophoraceae) chez la poule pondeuse, des graines de papaye (Carica papaya) chez l'aulacode (Thryonomys swinderianus Temminck, 1827) et des feuilles de Newbouldia laevis et de Zanthoxylum zanthoxylö̈des sur les nématodes parasites gastro-intestinaux des ovins Djallonké. Il a été observé par ailleurs une efficacité variant en fonction des molécules. Ainsi, l'Ivermectine et le Levamisole ont présenté une efficacité meilleure que celle de l'Albendazole. Cela serait en lien avec la disponibilité sur le marché local, le large spectre d'action et le prix relativement bas des Benzimidazoles (Albendazole) qui en font les produits les plus utilisés en élevage de petits ruminants dans la lutte contre les SGI en Côte d'Ivoire (Emanfo et al., 2015). En effet, plusieurs facteurs liés à la conduite de l'élevage peuvent entrainer l'apparition d'une résistance aux anthelminthiques dans les élevages. Il y a : (i) $\mathrm{La}$ fréquence d'utilisation de l'anthelminthique: certains schémas thérapeutiques comme ceux appliqués dans les élevages $\mathrm{n}^{\circ} 8$ et 11 , consistent à traiter les animaux toutes les 4, voire toutes les 3 semaines, dans le but d'empêcher la reproduction des parasites et ainsi assainir les pâtures. Cette approche conduit au remplacement progressif de tous les individus sensibles par des individus résistants, et assure la contamination des parcelles avec des larves exclusivement résistantes (Torres-Acosta et Hoste, 2008). Ce qui pourrait expliquer le trop grand nombre de vers adultes observé après autopsie dans ces élevages.

(ii) L'usage exclusif d'un anthelminthique, qui favoriserait également la pression de sélection sur les parasites, et conduirait ainsi inévitablement à l'installation d'une résistance contre tous les produits ayant le même mode d'action (Okombe, 2011). Par contre, l'usage alterné d'anthelminthiques de différents groupes pharmacologiques et présentant des mécanismes d'action différents ralentirait le développement de résistance (Menzies, 2010). Cette pratique de rotation a été observée dans l'élevage 4.

(iii) Le traitement systématique de l'ensemble des animaux du cheptel avant la mise à l'herbe sur une parcelle saine constitue aussi un facteur de risque clairement identifié (Cabaret, 2012). De tous les élevages, seul l'élevage 5 pratiquait le déparasitage sélectif. Une telle pratique est à encourager car elle pourrait expliquer l'efficacité des traitements avec l'absence de vers adultes dans le tube digestif des animaux de cette ferme après autopsie.

Les résultats des autopsies effectués dans chaque élevage ont révélé la présence de vers adultes dans le tube digestif des animaux des élevages où le pourcentage de réduction était inférieur à $100 \%$, confirmant ainsi les résultats de ces tests in vivo. Il faut tout de même signaler que dans certains élevages, les tests in vivo donnaient $100 \%$ de réduction pendant que l'autopsie révélait la présence de quelques vers adultes. Cela démontre la différence de sensibilité de ces approches : les tests in vivo, à eux seuls, ne sont pas suffisants pour confirmer l'absence de résistance. La 
résistance est détectée par les tests de laboratoire lorsque moins de 95\% des individus sont éliminés par le traitement ; à ce stade, au moins $25 \%$ des parasites sont déjà porteurs d'allèles de résistance (Paraud et al., 2014). Pour confirmer la suspicion de résistance, il faut rajouter à ces tests, d'autres tests plus précis comme la Polymerase Chain Reaction (PCR) qui détermine la résistance à partir de faibles pourcentages (mois de $5 \%$ ) de gènes de résistance détectés au sein de la population de parasite (Roeber et al., 2013 ; McNally et al., 2013 ; Jacquiet et al., 2014).

\section{Conclusion}

Cette étude a été réalisée pour confirmer la baisse de l'efficacité des traitements anthelminthiques en élevage ovin, déterminer les causes et proposer des solutions pour enrayer ce phénomène. Au terme de cette étude, il est à observer que les molécules utilisées restent encore efficaces de manière générale dans les élevages ovins du centre du pays. En effet, il existe une différence d'efficacité, avec l'Ivermectine qui a été testé dans un seul élevage et le Lévamisole qui apparaissent comme plus efficaces que l'Albendazole. Les résultats des tests menés lors de cette étude ne nous permettent pas d'affirmer la présence d'une résistance, ni d'une baisse de l'efficacité des anthelminthique utilisés. Mais il est tout de même important de souligner que si aucune mesure n'est prise pour y remédier, les pratiques des éleveurs telles que le non-respect des doses, le déparasitage massif et abusif du troupeau, l'absence de rotation de molécules pourraient conduire à l'installation d'une résistance à ces molécules. Cela entrainera la diminution des performances zootechniques des animaux et une perte économique directe pour l'éleveur. C'est pourquoi il est important de sensibiliser les éleveurs sur les conduites à tenir afin d'éviter ce fléau. La pratique du traitement ciblé sélectif qui n'est pas inconnue de certains éleveurs serait à encourager.

\section{CONFLIT D'INTERETS}

Les auteurs déclarent qu'ils n'ont pas de conflit d'intérêts par rapport à cet article.

\section{CONTRIBUTIONS DES AUTEURS}

AGAA est le principal investigateur. KEN et AMACK ont participé à la conception et la planification de l'étude ; GA a aidé à la collecte des données ; KEN a réalisé la révision critique du document; AMACK a aidé à la rédaction de la première version du manuscrit. KRA a aidé à la réalisation puis à l'interprétation des tests statistiques; AGAA a rédigé le document.

\section{REMERCIEMENTS}

Nous tenons à remercier tous les éleveurs et techniciens ayant contribué à la bonne conduite des travaux tant sur le terrain qu'au laboratoire.

\section{REFERENCES}

Achi YL, Zinsstag J, Yeo N, Dea V, Dorchies $\mathrm{Ph}$. 2003. Epidémiologie des helminthoses des moutons et des chèvres dans la région des savanes du Nord de la Côte d'Ivoire. Revue Méd. Vét., 154 : 179-188.

Berrag B, Cabaret J. 1998. Gastro-intestinal and pulmonary nematode infections decrease goat productivity in Morroccan semi-arid conditions. J. Helmint., 72 : 1520.

DOI: https://doi.org/10.1017/S0022149X 00000912

Boulkaboul A, Moulaye K, 2006. Parasitisme interne du mouton de race Ouled Djellal en zone semi-aride d'Algérie. Rev. Elev. Méd. Vét. Pays Trop., 59 : 23-29. DOI: https://doi.org/10.19182/remvt.9950

Cabaret J, 2012. Résistance des strongles aux anthelminthiques chez les ruminants. Le p. Vét., 43: 8-13.

Coles GC, Bauer C, Borgasteede FHM, Geerts S, Klei TR, Taylor MA, Waller PJ. 1992. World Association for the Advancement of Veterinary Parasitology (WAAVP) methods for detection of anthelmintic resistance in nematodes of veterinary importance. Vet. Parasitol., 44 : 35-44. DOI : $\quad$ https://doi.org/10.1016/03044017(92)90141-U

Dash K, Hall K, Barger IA, 1988. The role of arithmetic and geometric worm egg 
counts in faecal egg count reduction test and in monitoring strategic drenching programs in sheep. Aust. Vet. J., 65 : 6668. DOI: https://doi.org/10.1111/j.17510813.1988.tb07359.x .

Eichstadt M. 2017. Evaluation de la résistance des strongles gastro-intestinaux aux anthelminthiques dans quatre élevages ovins allaitants de Corrèze. PhD Thesis, Ecole nationale vétérinaire de Toulouse, France, $163 \mathrm{p}$.

Emanfo ASA, Esmel ME, Komoin-Oka AMAC, Kone MW. 2015. Health prophylaxis in goat breeding in southern and central Côte d'Ivoire. Bull. Anim. Hlth. Prod. Afr., 63: 505-517.

FAO (Organisation des Nations Unies pour l'alimentation et l'Agriculture). 2019. Données statistiques de la FAO, http://www.fao.org/faostat/fr/\#data/QA (consulté le 23/10/2019).

Jacquiet P, Barillet F, Bouix J, François D, Moreno C, Terefe G. 2009. La résistance génétique des ovins aux strongles gastrointestinaux. Bull. Acad. Vét. France, 162: 39-46. DOI : https://doi.org/10.4267/2042/47974

Kaplan RM. 2004. Drug resistance in nematodes of veterinary importance: a status report. Trends in Parasitol., 20 : 477-481.

DOI : https://doi.org/10.1016/j.pt.2004.08.001

Kochapakdee S, Pandey VS, Pralomkann W, Choldumrongkul S, Ngampongsai W, Lawpetchara A. 1995. Anthelmintic resistance in goats in Southern Thailand. Vet. Rec., 137: 124-125. DOI : http://dx.doi.org/10.1136/vr.137.5.124

Konaté S, Kampmann D. 2010. Atlas de la Biodiversité de l'Afrique de l'Ouest, Tome III. Abidjan \& Frankfurt/Main.

Kouakou SK, Toure A, Ouattara K, N'Guessan JD. 2010. Activité anticoccidienne in vivo de l'extrait aqueux des inflorescences de Thonningia sanguinea (Balanophoraceae) chez la poule pondeuse. Int. J. Biol. Chem. Sci., 4(4): 864-870. DOI: 10.4314/ijbcs.v4i4.62969

Mcnally J, Callan D, Andronicos N, Bott N, Hunt PW. 2013. DNA-based methodology for the quantification of gastrointestinal nematode eggs in sheep faeces. Vet. Parasitol., 198: 325-335. DOI :

https://doi.org/10.1016/j.vetpar.2013.09. 014

Menzies P. 2010. Manuel de Lutte contre les Parasites Internes du Mouton. University of Guelph press : Canada.

Okombe EV. 2011. Activité antihelminthique de la poudre d'écorce de racine de Vitex thomasii De Wild (Verbenaceae) sur Haemonchus contortus chez la chèvre. $\mathrm{PhD}$ Thesis, Université de Lubumbashi, R D Congo, p. 242.

Okombe EV, Pongombo SEW. 2013. Suspicion de la résistance aux benzimidazoles chez les strongles gastrointestinaux du caprin à Lubumbashi, R D Congo. Int. J. Biol. Chem. Sci., 6(7): 2426-2433. DOI : http://dx.doi.org/10.4314/ijbcs.v7i6.20

Paraud C, Pors I, Marcotty T, Devos I. 2014. Un premier cas de résistance aux lactones macrocycliques chez les nématodes gastrointestinaux confirmé en élevage ovin en France. Journée des Rencontres autour des Recherches sur les Ruminants, 21 : 3325-328.

Powers K, Wood L, Eckert J, Gibson T, Smith H. 1982. Guidelines for evaluating the efficacy of anthelminthics in ruminants (bovine and ovine). Vet. Parasitol., 10 : 205-264.

DOI : https://doi.org/10.1016/03044017(82)90078-4

Roeber F, Jex AR, Gasser RB. 2013. Impact of gastrointestinal parasitic nematodes of sheep, and the role of advanced molecular tools for exploring epidemiology and drug resistance-an Australian perspective. Paras. Vect., 6: 153. DOI: https://doi.org/10.1186/1756-3305-6-153

Sacramento TI, Ategbo J-M, Mensah GA, Adote-Hounzangbe S. 2010. Effet antiparasitaire des graines de papaye (Carica papaya) chez l'aulacode (Thryonomys swinderianus Temminck, 1827) d'élevage : cas des aulacodicultures du Sud-Bénin. Int. J. 
Biol. Chem. Sci., 4 (6): 2280-2293. DOI : 10.4314/ijbcs.v4i6.64981.

Sidi IYMS, Azando EVB, Olounlade PA, Hounzangbe-Adote MS. 2015. Effets combinés des feuilles de Newbouldia laevis et de Zanthoxylum zanthoxyloïdes sur les nématodes parasites gastrointestinaux des ovins Djallonké. Int. J. Biol. Chem. Sci., 9: 2078-2090. DOI : 10.4314/ijbcs.v9i4.30.

Tanguy I. 2011. Evaluation de la résistance des strongles digestifs aux anthelminthiques dans les élevages ovins en Bretagne. PhD Thesis, école nationale vétérinaire d'alfort, grande brétagne, $73 \mathrm{pp}$.

Touré G, Komoin-Oka C, Cabaret J, Ouattara Z, Faye B, Lhostis M. 2006. Peri-urban sheep breeding in Ivory Coast: evaluation of gastro-intestinal parasitism and influent factors of variation. J. Agr. Environ. Int. Dev., 100 : 155-168.

Torres-Acosta JFJ, Hoste H. 2008. Alternative or improved methods to limit gastrointestinal parasitism in grazing sheep and goats. Small Ruminant Research, 77 : 159-173. DOI : https://doi.org/10.1016/j.smallrumres.20 08.03.009

Zouh-Bi ZF, Touré A, Komoin C, Coulibaly M, Fantodji A. 2013. Parasites gastrointestinaux de l'aulacode (Thryonomys swinderianus, Temminck, 1827) au sud de la Côte d'Ivoire. Rev. Méd. Vét., 164(6): 312-318.

Zouiten H. 2006. Résistance aux anthelminthiques des nématodes parasites du tube digestif chez les ovins et les équidés au Maroc. PhD Thesis, université mohammed v - agdal, Maroc, $141 \mathrm{pp}$. 